\title{
Conjunctival Chemosis and Annular Ciliochoroidal Detachments Detected by Anterior-Segment Optical Coherence Tomography in a Case of Systemic Lupus Erythematosus
}

\author{
Akiko Ishida $^{a} \quad$ Yukari Yamane $^{a} \quad$ Yasurou Koyama $^{a} \quad$ Manabu Honda $^{b}$ \\ Masahiro Kondo $^{b}$ Yohko Murakawa ${ }^{b}$ Masaki Tanito $^{a}$ \\ aDepartment of Ophthalmology, Shimane University Faculty of Medicine, Izumo, Japan; \\ ${ }^{b}$ Department of Rheumatology, Shimane University Faculty of Medicine, Izumo, Japan
}

\section{Keywords}

Systemic lupus erythematosus · Lymphangiectasia - Ciliary detachment · Choroidal effusion · Anterior-segment optical coherence tomography

\begin{abstract}
A 61-year-old Japanese woman presented to our hospital for treatment of systemic serositis associated with systemic lupus erythematosus (SLE). At the initial ophthalmologic examination, her best-corrected visual acuity was 1.2 and 0.6 in her right and left eyes, respectively. Slit-lamp examination showed marked chemosis in both eyes (OU). Swept source-based, anterior-segment optical coherence tomography (AS-OCT) clearly showed conjunctival elevations corresponding to the chemosis in all scan directions OU. In some scans, hyporeflective spaces with luminal structures corresponding to dilated lymphatic channels and nonluminal structures corresponding to interstitial fluid accumulation were seen clearly under the conjunctival epithelium and/or in the parenchyma. In all scan directions, the supraciliary space was seen clearly, suggesting the presence of an annular ciliochoroidal detachment. Fundus examinations showed retinal edema temporal to the optic nerve head and subfoveal serous retinal detachments OU. Ocular effusions resolved by 2 weeks after the start of steroid pulse therapy, and pleural effusions and ascites resolved and pericardial effusion decreased by 2 months. AS-OCT can be useful for understanding the mechanism(s) of the less common anterior-segment ocular manifestations of SLE.
\end{abstract}




\section{Introduction}

Systemic lupus erythematosus (SLE) is a systemic autoimmune inflammatory disease caused by tissue depositions of immune complexes on the vascular wall. The ocular manifestations of SLE include retinopathy, optic neuropathy, choroidopathy, scleritis, episcleritis, and anterior uveitis; the retina is by far the site of the most frequently reported ocular lesions associated with SLE [1]. Less common ocular manifestations, conjunctival chemosis [2, 3], and choroidal detachment [4] have been reported. We report a patient with SLE in whom conjunctival chemosis and ciliary detachment were assessed using swept source-based, anterior-segment optical coherence tomography (AS-OCT).

\section{Case Report}

A 61-year-old Japanese woman reported fever and finger joint pain for 1 month. A local rheumatologist diagnosed SLE based on high levels of blood autoantibodies including antinuclear antibody (640×, speckled [normal range, $<40 \times])$, anti-double-strand DNA antibody $(7 \mathrm{IU} / \mathrm{mL}[\leq 6 \mathrm{IU} / \mathrm{mL}])$, anti-Smith antibody ( $\geq 600 \mathrm{U} / \mathrm{mL}[<10 \mathrm{U} / \mathrm{mL}])$, anti-ribonucleoprotein antibody (222 U/mL [ $<10 \mathrm{U} / \mathrm{mL}])$, anti-Ro (SS-A) antibody (19.6 U/mL [ $<10 \mathrm{U} / \mathrm{mL}])$, and low levels of serum complement titer and white blood cell counts. She fulfilled the 2019 European League Against Rheumatism/American College of Rheumatology Classification Criteria for SLE. Four months later, additional symptoms developed that included facial edema, malaise, and increased body weight (3-kg gain in a week). Radiologic and echographic examinations showed pleural and pericardial effusions and ascites; she was referred to the rheumatology department of our hospital for treatment of serositis associated with SLE. Since the serositis was refractory to oral prednisolone ( $30 \mathrm{mg} /$ day), steroid pulse therapy was planned and use of hydroxychloroquine was considered. She was referred to the ophthalmology department for the pre-dose consultation.

At the initial visit to the ophthalmology department, her best-corrected visual acuity was 1.2 in her right eye (OD) and 0.6 in her left eye (OS); the intraocular pressures were $12 \mathrm{~mm}$ $\mathrm{Hg}$ in both eyes (OU). Slit-lamp examination showed marked chemosis OU (Fig. 1a, b). An intraocular lens was fixed in the bag OU; no anterior chamber shallowing and cells were seen OU. AS-OCT (CASIA 2, Tomey Corporation, Nagoya, Japan) clearly showed conjunctival elevations corresponding to chemosis in all scan directions OU (Fig. 1c-j). Hyporeflective spaces with luminal structures (arrowheads) were seen in some scans corresponding to dilated lymphatic channels [5, 6], and nonluminal structures (asterisks) were seen corresponding to interstitial fluid accumulation under the conjunctival epithelium and/or in the parenchyma. The supraciliary spaces (blue arrows) were clearly depicted in all scan directions, suggesting the presence of an annular ciliochoroidal detachment. Color fundus photographs did not show signs of retinopathy, such as retinal hemorrhages or cotton-wool spots, OU (Fig. 2a, b). Macular OCT scans (RS3000 Advance 2, Nidek, Gamagori, Japan) showed retinal edema temporal to the optic nerve head (white arrows) and serous retinal detachments at the fovea (red arrows) OU (Fig. 2c, d). Fluorescein angiography (Heidelberg retina angiograph 2, Heidelberg Engineering, Dossenheim, Germany) showed areas of hyperfluorescence corresponding to the retinal edema at the temporal edge of the optic nerve head (white arrows) OU (Fig. 2e, f). Indocyanine green angiography (Heidelberg retina angiograph 2) showed areas of hyperfluorescence corresponding to serous retinal detachments in the foveal region (red arrows) OU (Fig. 2g, h).

The patient was not treated with hydroxychloroquine because of the presence of its contraindication (i.e., retinal pathology) but was treated with a steroid pulse regimen

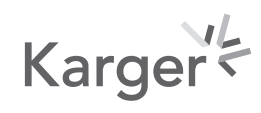


Fig. 1. Anterior-segment findings at the initial ophthalmic evaluation. A slitlamp examination shows marked chemosis in the right (a) and left (b) eyes. AS-OCT clearly shows conjunctival elevation and ciliochoroidal detachments (blue arrows) in all scan directions in the right (c, e, $\mathbf{g}, \mathbf{i})$ and left $(\mathbf{d}, \mathbf{f}, \mathbf{h}, \mathbf{j})$ eyes. In some scans, dilated lymphatic channels (arrowheads) and fluid space (asterisks) are seen clearly in the thickened conjunctiva. The red arrows in insets indicate the direction of the ASOCT scans. The panels shown are scans at nasal (c, f), temporal (d, e), superior $(\mathbf{g}, \mathbf{h})$, and inferior $(\mathbf{i}, \mathbf{j})$ part of the bulbar conjunctiva. AS-OCT, anterior-segment optical coherence tomography.

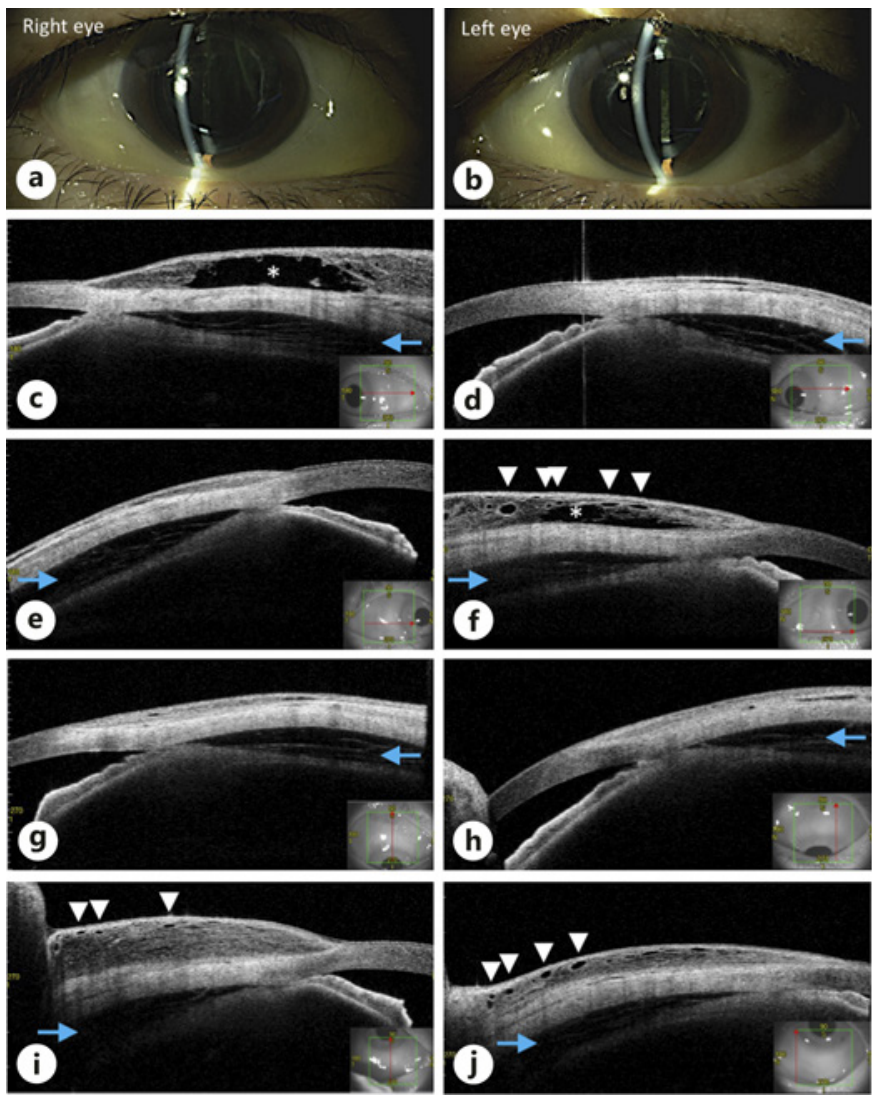

(methylprednisolone $500 \mathrm{mg} /$ day for 3 days) followed by oral prednisolone (25 mg/day) and tapering. Two weeks after the start of the steroid pulse therapy, the best-corrected visual acuities were $1.2 \mathrm{OD}$ and $0.9 \mathrm{OS}$ and the intraocular pressure levels were $9 \mathrm{~mm} \mathrm{Hg}$ $\mathrm{OD}$ and $8 \mathrm{~mm} \mathrm{Hg}$ OS. No chemosis was seen OU during a slit-lamp examination; the conjunctival elevation, dilated lymphatic channels, subconjunctival fluid accumulation, and ciliochoroidal detachments resolved based on the AS-OCT findings; and the retinal edema and serous retinal detachment resolved on the macular OCT scans. Two months after the start of the steroid pulse therapy, the pleural effusions and ascites resolved and the pericardial effusion decreased substantially.

\section{Discussion/Conclusion}

The current patient presented with hypoalbuminemia (2.8 g/dL [4.1-5.1 g/dL]); a renal biopsy showed the presence of class III lupus nephritis. Both previous SLE cases with chemosis [2] and severe choroidal detachment [4] had accompanied hypoalbuminemia. Accordingly, reduced osmotic pressure inside the vascular lumen of the conjunctival, uveal, and retinal vessels can explain the chemosis, ciliary detachment, and retinal edema/detachment in this case. However, despite resolution of these ocular effusions, the serum albumin level remained low $(3.0 \mathrm{~g} / \mathrm{dL})$, suggesting a mechanism(s) of effusion other than hypoproteinemia. No medication that possibly associated with conjunctival chemosis or macular edema was found in this case. 
Fig. 2. Fundus findings at the initial ophthalmic evaluation. The color fundus photographs show no signs of retinopathy such as retinal hemorrhages and cotton-wool spots in the right (a) and left (b) eyes. Macular optical coherence tomography scans show retinal edema temporal to the optic nerve head (white arrows) and serous retinal detachments at the fovea (red arrows) in the right (c) and left (d) eyes. Fluorescein angiography shows areas of hyperfluorescence corresponding to the retinal edema at the temporal edge of the optic nerve head (white arrows) in the right (e) and left (f) eyes. Indocyanine green angiography shows areas of hyperfluorescence corresponding to the serous retinal detachments in the foveal region (red arrows) in the right (g) and left (h) eyes.
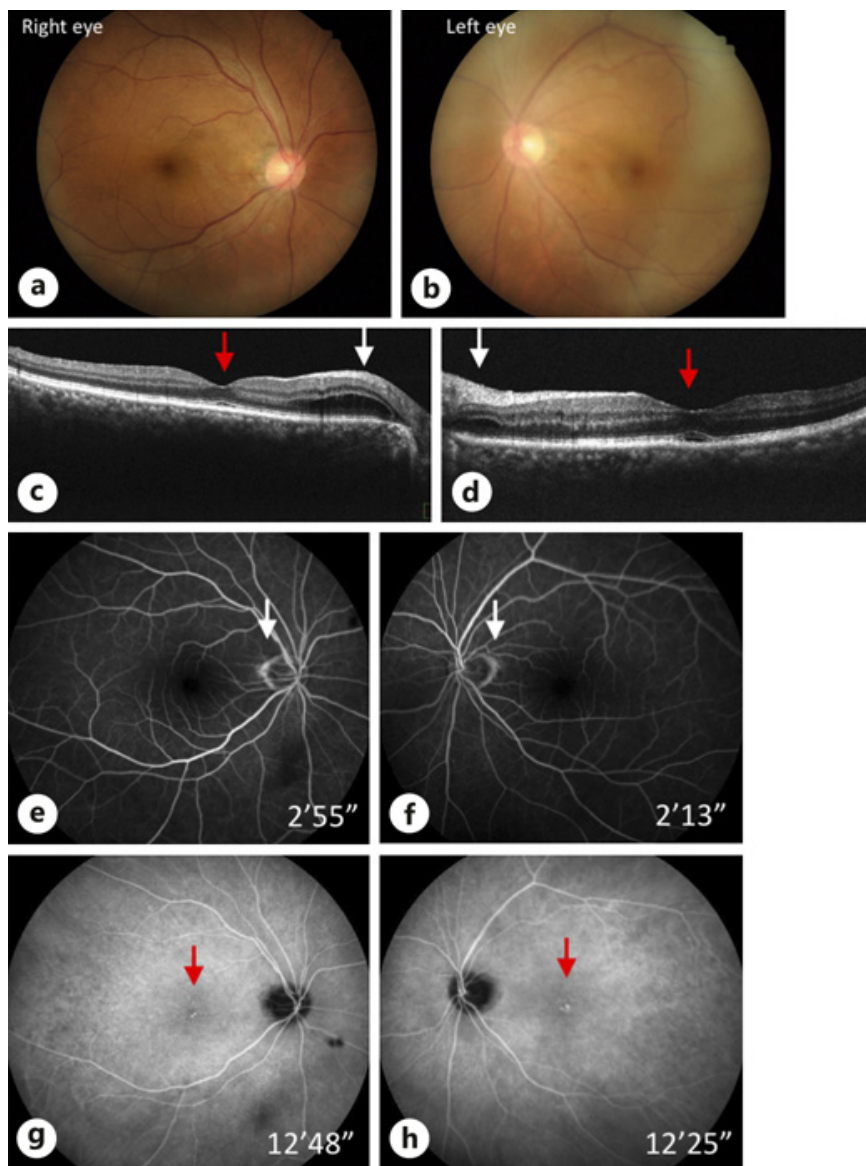

Previous reports have described deposition of immune complex in the blood vessel walls of the conjunctiva, ciliary body, retina, choroid, and sclera [7-9], which seems to correspond to the dilated lymphatic channels in the conjunctiva and ciliary/retinal effusions in the current case. Diffuse deposits also were found in association with the basement membranes in the bulbar conjunctiva, ciliary processes, and Bowman's layer in the cornea [7-9]. Thus, these extravascular depositions might explain the parenchymal accumulation of fluid in the conjunctiva in the current case. Both previous SLE cases with chemosis [2] and severe choroidal detachment [4] had accompanied pleural effusions. The multiple ocular effusions in the current case accompanied the signs of systemic serositis including pleuritis, pericarditis, and peritonitis; these ocular and systemic effusions responded to the steroid pulse therapy. Collectively, inflammatory reactions are the most plausible cause of the ocular manifestations in the case under discussion.

AS-OCT clearly demonstrated the presence of annular ciliochoroidal detachments that had been suggested but not directly detected in previously reported SLE cases with a myopic shift [3] or shallow anterior chamber [10]. A long-wavelength light source of the AS-OCT device and deeper penetration with swept source principle $[11,12]$ might be associated with the clear detection of ciliochoroidal detachments in our case. Previously, using AS-OCT, enlarged lymphatic channels were seen in cases with conjunctival lymphangiectasia, a rare condition involving the conjunctiva $[5,6]$. To the best of our knowledge, AS-OCT assessment of conjunctival chemosis and ciliary detachment in a case of SLE is unique in the literature. AS-OCT can be useful for understanding the mechanism(s) of these less common ocular manifestations of SLE. 


\section{Statement of Ethics}

This study adhered to the tenets of the Declaration of Helsinki. The patient provided written informed consent for publication of this case report and any accompanying pictures.

\section{Conflict of Interest Statement}

The authors have no conflicts of interest associated with this report.

\section{Funding Sources}

No financial support was provided.

\section{Author Contributions}

A.I., Y.Y., Y.K., M.H., M.K., Y.M., and M.T. treated the subject and collected the clinical data. A.I., Y.Y., and M.T. wrote the manuscript, and Y.K., M.H., M.K., and Y.M. revised the manuscript. All authors approved the final version of the manuscript. The authors are responsible for all aspects of this work.

\section{References}

1 Dammacco R. Systemic lupus erythematosus and ocular involvement: an overview. Clin Exp Med. 2018;18(2): 135-49.

2 Peponis V, Chalkiadakis S, Ergin S, Kyttaris VC. Chemosis as a presenting symptom of systemic lupus erythematosus. Lupus. 2010;19(8):997-1001.

3 Shu U, Takeuchi F, Tanimoto K, Moroi Y, Uchida K, Ito K. Transient myopia with severe chemosis associated with exacerbation of disease activity in systemic lupus erythematosus. J Rheumatol. 1992;19(2):297-301.

4 Furukawa Y., Yokoyama S., Tanaka Y., Kodera M., Kaga T.. A case of severe choroidal detachment in both eyes due to systemic lupus erythematosus. Am J Opthalmol Case Rep. 2020;19:100829.

5 Volek E, Toth J, Nagy ZZ, Schneider M. Evaluation of lymphatic vessel dilatations by anterior segment sweptsource optical coherence tomography: case report. BMC Ophthalmol. 2017;17(1):194.

6 Gokhale NS. AS-OCT in diffuse conjunctival lymphangiectasia. Indian J Ophthalmol. 2019;67(8):1338.

7 Aronson AJ, Ordoñez NG, Diddie KR, Ernest JT. Immune-complex deposition in the eye in systemic lupus erythematosus. Arch Intern Med. 1979;139(11):1312-3.

8 Karpik AG, Schwartz MM, Dickey LE, Streeten BW, Roberts JL. Ocular immune reactants in patients dying with systemic lupus erythematosus. Clin Immunol Immunopathol. 1985;35(3):295-312.

9 Frith P, Burge SM, Millard PR, Wojnarowska F. External ocular findings in lupus erythematosus: a clinical and immunopathological study. Br J Ophthalmol. 1990;74(3):163-7.

10 Stefater JA, Eliott D, Kim LA. Drainage and analysis of suprachoroidal fluid in a patient with acute systemic lupus erythematous. Am J Ophthalmol Case Rep. 2017;5:29-32.

11 Shoji T, Kato N, Ishikawa S, Ibuki H, Yamada N, Kimura I, et al. In vivo crystalline lens measurements with novel swept-source optical coherent tomography: an investigation on variability of measurement. BMJ Open Ophthalmol. 2017;1(1):e000058.

12 Xu BY, Mai DD, Penteado RC, Saunders L, Weinreb RN. Reproducibility and agreement of anterior segment parameter measurements obtained using the CASIA2 and spectralis OCT2 optical coherence tomography devices. J Glaucoma. 2017;26(11):974-9. 